\title{
Lessons learnt from the emergence of Zika virus
}

\author{
Looking back at how Zika virus emergence was handled during and after the 2015-2016 outbreak will be important \\ for assessing how well multiple relevant stakeholders were integrated to mount a response, and can provide the \\ groundwork to better cope with emerging infections in the future.
}

\section{Marc Lecuit and Laurent Nguyen}

\section{7} ika virus (ZIKV) is an arthropodborne flavivirus that was first isolated in 1947 in a sentinel rhesus monkey from the Zika forest in Uganda, in the context of yellow fever surveillance. It is mainly transmitted to humans by Aedes aegypti, a mosquito of tropical and subtropical areas that is also the vector of other major flaviviruses, such as Dengue virus. Until recently, ZIKV had only been associated with sporadic cases of benign human infection in Africa and Asia that were characterized by fever and rash $^{1}$. The virus emerged a decade ago in the South Pacific, when the first outbreak was reported in 2007 in Micronesia $^{2}$. It was later responsible for a larger outbreak in French Polynesia in 2013, but its association with microcephaly was only suspected when it reached Latin America ${ }^{3}$. This suspicion was rapidly supported epidemiologically by a retrospective study conducted in French Polynesia, and proven by prospective studies in Brazil and the Caribbean ${ }^{4}$. More than a million people were infected in total between 2015 and 2016 (ref. ${ }^{5}$ ). It was later shown that ZIKV infection in pregnant women is teratogenic and associated with severe birth defects, including ocular defects and profound congenital microcephaly cases in Brazil ${ }^{6,7}$. The risk of developing congenital microcephaly, as recognized in Brazil in January 2016, is higher if the infection occurs during the first trimester of pregnancy and was globally estimated to affect approximately $5 \%$ of fetuses and infants born to ZIKV-infected mothers ${ }^{8,9}$. In addition, a retrospective epidemiological study conducted in French Polynesia led to the discovery of its possible association with Guillain-Barré syndrome ${ }^{10}$.

Contextual fostering of ZIKV research The general media reported intensively on the ZIKV epidemic in Brazil. The extensive coverage and the consequences of infection for human health, including an unprecedented epidemic of congenital microcephaly in neonates, contributed to the growing worldwide concern. Indeed, the World Health Organization (WHO) declared in February 2016 that ZIKV disease was a "Public Health Emergency of International Concern" ${ }^{11}$. Pressures including the heightened public awareness, as well as calls from scientific and public health experts, triggered the political will to dedicate substantial strategic funds to foster research on ZIKV. As an example, the European Union has invested $€ 45$ million in ZIKV research ${ }^{12}$. The need to resolve this international health crisis was felt as a moral duty among many, prompting collaborative efforts between clinicians and researchers to identify and tackle ZIKV-associated pathologies. In addition to its major public health importance, ZIKV research was also driven by scientific curiosity to understand how ZIKV could induce such pathologies, which have never previously described for similar viruses. Research efforts advanced quickly, in particular in characterizing the congenital-associated syndrome associated with ZIKV infection, and their underlying molecular mechanisms ${ }^{13,14}$.

Research into ZIKV emergence, spread and pathogenesis also benefitted from existing infrastructure and previous knowledge of related infections. For example, epidemiological and medical research used existing surveillance programmes for vertically transmitted infections, and registries that monitor fetal abnormalities. On the scientific side, ZIKV research built on work with cell and animal models (rodents and non-human primates) and clinical cohorts to study recently emerged arboviruses, such as chikungunya virus, and other flaviviruses (for example, Dengue, West Nile virus, yellow fever virus) (reviewed in ref. ${ }^{15}$ ), and pathogens associated with congenital pathologies (TORCH: toxoplasmosis, others (syphilis, varicella, mumps, parvovirus B19 and HIV), rubella, cytomegalovirus, herpes simplex virus). Major advances in modelling ZIKV-induced congenital microcephaly were also facilitated by prior understanding of fundamental biological processes, such as brain development, and existing mouse models to study them ${ }^{16}$. Recent advances in human neural stem cell biology and technical developments to generate two- and three-dimensional human in vitro culture models have been instrumental in beginning to decipher the underlying mechanisms of ZIKV-associated neuropathology ${ }^{17-20}$. More research is now dedicated to understanding the possible longstanding consequences of congenital ZIKV infection on brain maturation and the acquisition of adapted motor and social behaviours.

Clinical diagnosis of ZIKV infection is not straightforward as most of its symptoms overlap with those of other arbovirus infections, but serological and RT-PCR assays allow rapid diagnosis and were deployed in the field during the outbreak ${ }^{21,22}$. Considerable efforts have also been devoted to identify effective cures and preventive measures for ZIKV infection. Several potentially useful drugs have been identified in preclinical testing and viable vaccine candidates are advancing quickly through the different discovery phases ${ }^{21,23,24}$.

\section{Outstanding questions}

ZIKV has been shown to cross the placenta and infect the fetus, leading to major pathology. This vertical transmission and the resulting effects on the developing fetus were uncovered several decades after its first isolation in Uganda when the virus emerged and spread in highly medicalized areas such as French Polynesia, Brazil and the Caribbean, where national surveillance systems monitoring birth defects and unusual conditions are implemented. It is unclear whether the emergence of congenital ZIKV syndrome is owed mainly to greater surveillance, or whether mutations acquired by ZIKV during its recent spread have also increased infectivity and pathogenesis ${ }^{25}$.

While ZIKV is primarily transmitted by the Aedes mosquito bite, it has also been reported to be transmitted by blood transfusion and possibly breastfeeding. Importantly, detailed investigations of imported cases of ZIKV in areas where known vectors are absent also led to the identification of unexpected cases of sexual transmission ${ }^{26,27}$, a first for an arbovirus. Indeed, circumstantial evidence obtained 
Box 1 | Outstanding questions regarding ZIKV pathogenesis

(1) What are the characteristics of ZIKV systemic infection?

(2) What are the determinants of maternal ZIKV viraemia?

(3) How does ZIKV cross the placental barrier?

(4) How does ZIKV cross the bloodbrain barrier?

(5) What are the mechanisms of ZIKV dissemination within the brain?

(6) Does ZIKV persist in the brain and other tissues after birth?

(7) Which cells are infected with ZIKV in the brain?

(8) What is the pathogenesis of ZIKVassociated Guillain-Barré syndrome?

as early as 2011 pointed to a possible nonvector-mediated transmission route for ZIKV, when a patient infected by ZIKV in Africa subsequently infected his wife in United States. The transmission of ZIKV by sexual contact remains poorly understood but is fully supported by epidemiological reports and experimental data obtained in animal model ${ }^{28,29}$, which suggests that persistence of ZIKV in genital fluid favours sexual transmission ${ }^{30}$, although exactly how this is achieved remains unclear.

The pace of discovery has been remarkable; it took an impressively short time to move from the identification of ZIKV emergence in Latin America to the demonstration that it was transmitted via transplacental route to the developing fetus, and subsequently to where it infects cortical neurons and their progenitor to interfere with cerebral cortex development. However, many outstanding questions remain to be answered, regarding both ZIKV pathogenesis (Box 1) and the effect of co-infections, notably with other arboviruses that are known to co-circulate in the same regions and co-transmit via the mosquito vectors $^{31}$. While convergent studies conducted in animal and human models demonstrated that ZIKV replicates in the placenta $^{32,33}$, the in vivo mechanisms of placental infection remain elusive. Moreover, once in the placenta, ZIKV can replicate in Hofbauer cells ${ }^{32,34}$, but the mechanism and significance of Hofbauer infection for ZIKV pathogenesis remains unknown. More research is also needed to understand how ZIKV accesses the central nervous system of the fetus. Is an active mechanism involved in blood-brain barrier crossing, or does the immaturity of the blood-brain barrier allow a passive transfer of ZIKV in the developing brain? Are microglia disseminating ZIKV in vivo in the developing brain ${ }^{35}$ ? Another unanswered question relates to the selective tropism that ZIKV shows for brain stem cells $^{13,36,37}$. It is still unclear how ZIKV reaches these cells in vivo and whether they are the initial cellular target following ZIKV entry to the developing brain. Moreover, as ZIKV interferes with vessel development ${ }^{38}$, it would be interesting to assess the impact of cell-autonomous and non-cellautonomous responses on ZIKV cellular pathology. Finally, as has been reported for other teratogenic viruses associated with brain malformation (for example, rubella virus), ZIKV may persist after birth in the brain of in-utero-infected children, leading to additional postnatal insults that would add another layer of complexity to interpret ZIKV-induced neurological deficits. An additional key topic is the identification of the host and viral factors implicated in ZIKV-associated Guillain-Barré syndrome, which is a severe neurological complication. Although the ZIKV outbreak is now over in Latin America, answering these outstanding scientific questions will help to better prepare us for probable future re-emergences of ZIKV.

\section{Perspectives on the response}

Reviewing how the ZIKV outbreak was handled, a few crucial steps emerge that need to be addressed rapidly and precisely in the event of a new outbreak. Firstly, identifying the emergence and associated clinical phenotypes of a new outbreak rapidly and accurately is of utmost importance, underlining the critical need for effective registries and surveillance systems, and the need to compile detailed clinical and biological assessment of infected cases as early as possible as an emergence unfolds. Secondly, the modes of pathogen transmission need to be rapidly identified to understand the magnitude and ramifications of the threat, which requires rapid implementation of descriptive epidemiological and case-control studies. Thirdly, researchers need to identify basic scientific questions to be addressed, which include deciphering the basic microbiology of the emerging microorganism, and the development of diagnostic and research tools to characterize the pathogen, the delineation of the biological phenotypes associated with the infection and their underlying mechanisms, which may help develop countermeasures aimed at stopping the emerging microorganism and/or its impact on human health. This requires the development of experimental systems that allow faithful reproduction of the infection characteristics, including cell, tissue and animal models. Their relevance will need to be assessed by comparison to human data.

Rapid progress in these areas requires close collaboration between clinicians and scientists, as well as mutual understanding of each other's needs and priorities.

Working with local communities and empowering them to better respond to future threats is also essential to foster a collaborative environment and bring about true impact where outbreaks emerge. Indeed, some of the infrastructure put in place for diagnosis and surveillance during the Brazil ZIKV outbreak were subsequently used to respond more effectively to the recent yellow fever outbreak ${ }^{39}$.

Media coverage is useful in creating the necessary social and political awareness that precedes the release of funds to tackle an emerging outbreak, but should be measured and accurate to avoid causing unnecessary panic and distress in the population. The intense interest triggered by ZIKV emergence shook the conventional rules of scientific publishing. The initial rush to publish - aroused by international media attention and the legitimate need to rapidly disseminate new knowledge in this field - led many high-profile scientific journals to loosen their usual scientific scrutiny and publish reports that could be considered to be preliminary, with the risk of privileging 'novelty' over scientific rigor and slowing progress in the characterization of the emergent virus, and potentially discrediting ZIKV research in the eyes of researchers in other relevant fields. Maintaining high publication standards, to be aware of and to specifically discuss the limitations of experimental models, and to check for consistency between animals and human data before reaching conclusions, is critical. The recent development of preprint publishing services (such as bioRxiv) should allow a better conciliation of the apparent contradictory need to rapidly release research results of high relevance to public health, and allow publication in peer-reviewed journals to fully ensure their scientific rigor, consistency and relevance.

\footnotetext{
Marc Lecuit ${ }^{1,2,3 *}$ and Laurent Nguyen ${ }^{4 *}$ ${ }^{1}$ Institut Pasteur, Biology of Infection Unit, Paris, France. ${ }^{2}$ Inserm U1117, Paris, France. ${ }^{3}$ Paris Descartes University, Department of Infectious Diseases and Tropical Medicine, Necker-Enfants Malades University Hospital, Institut Imagine, Paris, France. ${ }^{4}$ GIGA-Neurosciences, Interdisciplinary Cluster for Applied Genoproteomics (GIGA-R), University of Liège, C.H.U. Sart Tilman, Liège, Belgium. *e-mail:marc.lecuit@pasteur.fr;.nnguyen@uliege.be
}

Published online: 24 August 2018

https://doi.org/10.1038/s41564-018-0233-4 
References

1. Macnamara, F. N. Trans. R. Soc. Trop. Med. Hyg. 48, 139-145 (1954).

2. Duffy, M. R. et al. N. Engl. J. Med. 360, 2536-2543 (2009).

3. Musso, D. et al. Euro Surveill. 19, 20761 (2014).

4. Cauchemez, S. et al. Lancet 387, 2125-2132 (2016).

5. Zika Cumulative Cases (PAHO, WHO, accessed 1 July 2018); https://go.nature.com/2tNktxY

6. Driggers, R. W. et al. N. Engl. J. Med. 374, 2142-2151 (2016).

7. Mlakar, J. et al. N. Engl. J. Med. 374, 951-958 (2016).

8. Hoen, B. et al. N. Engl. J. Med. 378, 985-994 (2018).

9. Honein, M. A. et al. JAMA 317, 59-68 (2017).

10. Cao-Lormeau, V. M. et al. Lancet 387, 1531-1539 (2016).

11. WHO statement on the first meeting of the International Health Regulations (2005) (IHR 2005) Emergency Committee on Zika virus and observed increase in neurological disorders and neonatal malformations. World Health Organization (1 February 2016); https://go.nature.com/2NiMKo2

12. Zika: Commission activities in the area of Zika (European

Commission, 2016); https://go.nature.com/2tRKyMb

13. Gladwyn-Ng, I. et al. Nat. Neurosci. 21, 63-71 (2018).

14. Xu, M. et al. Nat. Med. 22, 1101-1107 (2016).

15. Weaver, S. C., Charlier, C., Vasilakis, N. \& Lecuit, M. Annu. Rev Med. 69, 395-408 (2018).
16. Laguesse, S. et al. Dev. Cell 35, 553-567 (2015).

17. Dang, J. et al. Cell Stem Cell 19, 258-265 (2016).

18. Li, Y. et al. Cell Stem Cell 3, 385-396 (2016).

19. Qian, X. et al. Cell 165, 1238-1254 (2016).

20. Watanabe, M. et al. Cell. Rep. 21, 517-532 (2017).

21. Aliota, M. T. et al. Antiviral Res. 144, 223-246 (2017).

22. Corman, V. M. et al. Bull. World Health Organ. 94 880-892 (2016)

23. Poland, G. A. et al. Lancet Infect. Dis. 18, 211-219 (2018).

24. Subudhi, B. B., Chattopadhyay, S., Mishra, P. \& Kumar, A. Viruses 10, E235 (2018).

25. Yuan, L. et al. Science 358, 933-936 (2017).

26. Coelho, F. C. et al. Int. J. Infect. Dis. 51, 128-132 (2016).

27. McCarthy, M. BMJ 352, i720 (2016)

28. Duggal, N. K. et al. Cell. Rep. 18, 1751-1760 (2017).

29. Tang, W. W. et al. Cell. Rep. 17, 3091-3098 (2016).

30. Moreira, J., Peixoto, T. M., Siqueira, A. M. \& Lamas, C. C. Clin. Microbiol. Infect. 23, 296-305 (2017).

31. Moreira, J., Bressan, C. S., Brasil, P. \& Siqueira, A. M. Clin. Microbiol. Infect. 24, 827-835 (2018).

32. Martines, R. B. et al. Lancet 388, 898-904 (2016).

33. Miner, J. J. et al. Cell 165, 1081-1091 (2016).

34. Noronha, L., Zanluca, C., Azevedo, M. L., Luz, K. G. \& Santos, C. N. Mem. Inst. Oswaldo Cruz 111, 287-293 (2016).
35. Mesci, P. et al. Hum. Mol. Genet. 27, 41-52 (2018). 36. Chavali, P. L. et al. Science 357, 83-88 (2017). 37. Wu, K. Y. et al. Cell Res. 26, 645-654 (2016). 38. Shao, Q. et al. Development 143, 4127-4136 (2016).

39. Callender, D. M. Glob. Public Health http://doi.org/crpz (2018).

\section{Acknowledgements}

This work was supported by the European Union's

Horizon 2020 Research and Innovation Programme under ZIKAlliance Grant Agreement No. 734548 (to L.N. and M.L.). L.N. is senior research associate from F.R.S-F.N.R.S. and his research is also supported by F.R.S.F.N.R.S. (J.0163.16 and J.0028.18 - CDR), the Fonds Léon Fredericq, the Fondation Médicale Reine Elisabeth, the Fondation Simone et Pierre Clerdent, the Belgian Science Policy (IAP-VII network P7/20), the ARC (ARC11/16-01), and the ERANET Neuron STEM-MCD; M.L. is also funded by Institut Pasteur, Inserm and LabEx IBEID.

Competing interests

The authors declare no competing interests. 